\title{
Short-term effects of nitrate-rich green leafy vegetables on blood pressure and arterial stiffness in individuals with high-normal blood pressure
}

Catherine P Bondonno $\mathrm{PhD}^{\mathrm{a}^{*}}$, Alex H Liu ${ }^{\mathrm{a}^{*}}$, Kevin D Croft $\mathrm{PhD}^{\mathrm{a}}$, Natalie C Ward $\mathrm{PhD}^{\mathrm{a}}$, Xingbin Yang $\mathrm{PhD}^{\mathrm{b}}$, Michael J Considine $\mathrm{PhD}^{\mathrm{c}, \mathrm{d}}$, Ian B Puddey $\mathrm{MD}^{\mathrm{a}}$, Richard J Woodman $\mathrm{PhD}^{\mathrm{e}}$, Jonathan M Hodgson $\mathrm{PhD}^{\mathrm{a}}$

${ }^{a}$ School of Medicine and Pharmacology, University of Western Australia, Perth, WA, Australia

${ }^{b}$ College of Food Engineering and Nutritional Science, Shaanxi Normal University, Xi'an, China

${ }^{c}$ School of Plant Biology, University of Western Australia, Perth, WA, Australia

${ }^{d}$ Department of Agriculture and Food Western Australia, South Perth, WA, Australia

${ }^{e}$ Flinders Centre for Epidemiology and Biostatistics, Flinders University, Adelaide, SA, Australia

*These authors contributed equally to this work

\section{Address for correspondence and requests for reprints:}

Catherine P Bondonno

School of Medicine and Pharmacology

Level 3, Medical Research Foundation

Rear 50 Murray St, Perth Western Australia, Australia WA6000

GPO Box X2213 Perth Western Australia, Australia WA6847

Tel: 61892240342

Fax: 61892240246

Email: cbondonno@meddent.uwa.edu.au 


\section{ABSTRACT}

Evidence for a beneficial effect of dietary nitrate, through the nitrate-nitrite-NO pathway, on measures of cardiovascular function in healthy individuals is accumulating. It is less clear whether increased dietary nitrate intake from green leafy vegetables would have similar beneficial vascular effects in those at increased risk of developing hypertension. Our aim was to assess the effects of short term regular consumption of increased nitrate from green leafy vegetables on blood pressure and arterial stiffness in individuals with high-normal blood pressure. Thirty eight men and women aged 30-70 years with systolic blood pressure 120 to 139 mmHg were recruited to a randomised controlled cross-over trial. The effects of a 7 day high nitrate diet intervention (increased nitrate intake by at least $300 \mathrm{mg} /$ day from green leafy vegetables) were compared to a 7 day low nitrate diet intervention. Outcome measures included pre and post salivary and plasma nitrate and nitrite concentrations, ambulatory, home and office blood pressure, augmentation index and carotid-femoral pulse wave velocity. The high nitrate diet intervention resulted in at least a fourfold increase in salivary and plasma nitrate and nitrite $(\mathrm{P}<0.001)$. Ambulatory, home and office blood pressure and arterial stiffness were not different between the high nitrate diet and the low nitrate diet. Increasing dietary nitrate intake in those with high normal blood pressure may not be an effective short-term strategy to lower blood pressure in those at increased risk of hypertension.

KEYWORDS: nitrate, green leafy vegetables, blood pressure, arterial stiffness 


\section{Introduction}

The majority of cardiovascular disease risk factors, including high blood pressure, dyslipidemia and endothelial dysfunction, are associated with an unhealthy lifestyle and poor diet. These risk factors are modifiable, highlighting the critical importance of prevention strategies. One such strategy is a diet rich in fruit and vegetables. Increased intake of fruit and vegetables, in particular

green leafy and cruciferous vegetables, is associated with decreased cardiovascular risk [1, 2]. Green leafy vegetables, such as lettuce, rocket and spinach, are rich in nitrate [3]. Recent evidence suggests that dietary nitrate may be a cardioprotective component of a vegetable rich diet and that cardioprotection occurs via effects on the circulating pool of nitric oxide (NO) [4]. Dietary nitrate can enhance NO status through the enterosalivary nitrate-nitrite-NO pathway providing a physiologically relevant storage reservoir of bioactive NO [5]. Endothelial derived NO has a number of critical functions including effects on basal vascular tone, arterial stiffness and blood pressure.

Evidence for the beneficial effects of dietary nitrate, through the nitrate-nitrite-NO pathway, on measures of cardiovascular health in healthy individuals is accumulating. More than 25 clinical trials have examined the effect of an acute or chronic increase in nitrate intake on blood pressure and/or vascular function [6]. Most of these studies are, however, conducted with nitrate from beetroot juice or nitrate salts. Two acute studies have examined spinach intake $[7,8]$ while only one chronic study has examined increased intake of green leafy vegetables found in a Japanese traditional diet [9]. Evidence from clinical trials suggests a public health message to increase nitrate-rich green leafy vegetable intake could be an important strategy in cardiovascular disease prevention in healthy individuals. However, the vascular effect of chronic intake of nitrate rich green leafy vegetables commonly consumed in a Western diet has not yet been determined. Also 
unknown is whether the cardioprotective effect observed with dietary nitrate in healthy individuals will be observed in individuals with elevated risk for hypertension, such as those with high-normal range blood pressure.

The objective of this study was to assess the vascular effects of regular consumption (7 days) of increased dietary nitrate from green leafy vegetables commonly consumed in a Western diet in men and women with high-normal blood pressure. Vascular effects were determined by measuring blood pressure (ambulatory, home and office) and arterial stiffness (carotid-femoral pulse wave velocity (cfPWV) and augmentation index (AIx)). 


\section{Methods}

\section{Participants}

Participants $(n=38)$ were recruited by newspaper advertisement from the Perth general population. Prior to enrolment, participants were screened within the University of Western Australia, School of Medicine and Pharmacology Unit, located at Royal Perth Hospital. Screening consisted of a standard medical history questionnaire, blood pressure measurement, electrocardiography, height, weight and body mass index (BMI) measurement as well as routine laboratory analysis of a fasting blood sample. Exclusion criteria included: BMI $<18$ or $>37$

$\mathrm{kg} / \mathrm{m}^{2}$; age $<21$ y or $>75$ years; use of antihypertensive medication; systolic blood pressure $<120$ or $\geq 140 \mathrm{mmHg}$ or diastolic blood pressure $<65 \mathrm{~mm} \mathrm{Hg}$ or $\geq 90 \mathrm{mmHg}$; a diet high in green leafy vegetables; use of antibacterial mouth wash; use of antibiotics (within previous 2 months); current or recent (<12 months) smoking; history of cardiovascular or peripheral vascular disease; diagnosed type 1 or type 2 diabetes; recent history of a psychiatric illness or other major illnesses such as cancer; recent history of asthma, renal, liver, gastrointestinal disease or gout; a change in drug therapy within the previous 3 months, or the likelihood that drug therapy would change during the study; current or recent (within previous 6 months) significant weight loss or gain (> $6 \%$ of body weight); $>20 \mathrm{~g} / \mathrm{d}$ alcohol consumption; women who were pregnant, lactating or wishing to become pregnant during the study; and inability or unwillingness to consume foods provided in the trial. Participants were asked to refrain from the use of mouth wash from two weeks prior to their first study visit to the end of the study. The study was carried out in accordance with the Declaration of Helsinki and was approved by the University of Western Australia Human Research Ethics Committee. Participants provided written informed consent 
before inclusion in the study. The trial was registered with the Australian New Zealand Clinical Trials Registry (ACTRN12611000609954).

\section{Study design}

The study followed a randomised controlled cross over design. Participants were allocated to an intervention plan via block randomisation using computer-generated random numbers devised by a statistician. After a 7 day washout period, the study comprised two 7 day dietary intervention periods separated by a 7 day washout. Intervention periods comprised a low nitrate diet (nitrate intake less than $100 \mathrm{mg} /$ day) and a high nitrate diet (nitrate intake greater than $300 \mathrm{mg} /$ day). Throughout the study period, participants monitored their home blood pressure twice daily. Each participant completed a total of 4 visits to the School of Medicine and Pharmacology. All visits were at the same time of day. For each intervention period, these visits were scheduled at baseline and day 7. Adherence to study protocol was verified at each visit with a standard questionnaire. At all visits a plasma sample was taken for analysis of plasma nitrate, and nitrite, a saliva sample was taken for analysis of saliva nitrate and nitrite, blood pressure was measured and arterial stiffness measures comprising augmentation index (AIx) and carotid-femoral pulse wave velocity (cfPWV) were performed. A 24 hour urine sample for analysis of urinary sodium, potassium and creatinine levels was performed on day 7.

\section{Interventions}

The high nitrate diet intervention (with the objective to increase nitrate intake by at least 300 mg/day) was achieved via an increased intake of green leafy vegetables. Green leafy vegetables were provided as frozen spinach $(250 \mathrm{~g} / \mathrm{d})$, obtained from a single batch, and the rest supplied as fresh green salad vegetables consisting of lettuce, spinach, rocket and other leafy green 
vegetables (120g/d). For the low nitrate diet intervention (nitrate intake less than $100 \mathrm{mg} /$ day)

and washout periods, participants were instructed to avoid intake of vegetables rich in nitrate (including lettuce, beetroot, celery, spinach, Chinese greens, other leafy greens, parsley and related herbs) and to minimise intake of other green vegetables (including, broccoli, cabbage, brussels sprouts and cauliflower). A list of foods to avoid or limit was provided to each participant. The average dietary nitrate intake in Western populations is estimated to be approximately $100 \mathrm{mg} / \mathrm{d}$ [3]. Therefore, the change in diet for most participants was minimal. During two days of the high nitrate diet intervention period, participants were required to complete a food diary detailing their green leafy vegetable intake.

\section{Nitrate and nitrite analysis}

Levels of nitrate and nitrite were measured in the cooked spinach, saliva and plasma samples. Spinach samples were prepared by homogenising $50 \mathrm{~g}$ of spinach with $100 \mathrm{ml}$ of deionised distilled water for $5 \mathrm{~min}$. A $50 \mathrm{~g}$ sample of the homogenized spinach was then mixed with $50 \mathrm{ml}$ of deionised distilled water for $15 \mathrm{~min}$ at $55^{\circ} \mathrm{C}$. A $10 \mathrm{ml}$ sample was mixed with an equal volume of acetonitrile, centrifuged $\left(4^{\circ} \mathrm{C}\right)$ for $15 \mathrm{~min}$ at $3500 \mathrm{rpm}$, and the supernatant analysed for nitrate content. Blood samples were collected into EDTA tubes, immediately centrifuged (15 min at $4^{\circ} \mathrm{C}$ ) and plasma stored at $-80^{\circ} \mathrm{C}$ until measurement. A 5 minute saliva sample was collected and stored at $-80^{\circ} \mathrm{C}$ until measurement. Nitrate and nitrite concentrations in spinach, plasma and saliva samples were measured using gas chromatography-mass spectrometry (GC-MS [10]. To determine nitrite contamination from sampling procedures, deionised water was added to EDTA tubes and analysed as above. The measured nitrite contamination was $0.1 \mu \mathrm{mol} / \mathrm{L}$. Plasma nitrite measures were adjusted accordingly. 
Nitrate content of the fresh green leafy vegetables provided for the high nitrate diet intervention were estimated using mean literature values [11]. Estimates were based on the proportional weights of specific types of leafy green vegetables included in the salad mix.

\section{Blood pressure measurement}

Home blood pressure

During each 7d intervention period, participants measured their blood pressure in the morning (1 hour after breakfast) and the evening (1 hour after dinner) using an A\&D Medical UA-767PC digital blood pressure monitor (A\&D Instruments Ltd., Abingdon, Oxon OX14, U.K.). At each time point, 5 home blood pressure measurements were performed with a 1 minute interval between measurements. The first reading was not used in the analysis. Participants were instructed to rest for 5 minutes in a seated position before commencing blood pressure measurements. Day 1 morning reading was not included in the analysis as green leafy vegetable intake had not commenced at this time point.

\section{Ambulatory blood pressure}

A 10-h day time ambulatory blood pressure recording was performed at baseline and day 7 for each intervention period. Ambulatory blood pressure was assessed by a Spacelabs monitor (Spacelabs Medical Inc) fitted by a trained researcher to the non-dominant arm about $2.5 \mathrm{~cm}$ above the antecubital fossa. Blood pressure and heart rate were measured every 20 minutes. Participants were instructed to continue their usual daily activities and to avoid vigorous exercise. Measurements showing an error code or those with a pulse pressure of less than $20 \mathrm{~mm}$ Hg were 
excluded from the analysis. Blood pressure traces were considered complete if more than $80 \%$ of the recordings were valid.

Office blood pressure

A single office blood pressure measurement was performed prior to assessment of arterial stiffness. The blood pressure measurement was performed using a Dinamap 1846SX/P oscillometric recorder (Critikon, Tampa, FL, USA). Subjects rested for 2 min in a supine position, and blood pressure was then recorded.

\section{Arterial stiffness}

The carotid-femoral pulse wave velocity (cfPWV) and augmentation index (AIix) was measured at baseline and day 7 using the SphygmoCor pulse wave analysis system (AtCor Medical, Sydney, Australia. Model MM3. Software version 9) according to a previously published protocol [8].

\section{Urinary sodium, potassium and creatinine analysis}

Urinary sodium, potassium and creatinine were measured using routine biochemical analyses. Measurements were performed by the Department of Clinical Biochemistry at Royal Perth Hospital. Sodium and potassium concentrations were measured with the use of an ion-selective electrode with an automated analyzer (Roche Hitachi 917). Creatinine was measured using a kinetic colorimetric test (Roche, Indianapolis, IN) with an automated analyzer (Roche Hitachi 917).

\section{Other biochemical analyses}


Routine biochemical analyses were performed at screening in the PathWest laboratory at Royal Perth Hospital, Western Australia. Serum total cholesterol, HDL cholesterol and triglycerides were measured using a routine enzymatic colorimetric test with a fully automated analyser (Roche Hitachi 917, Roche Diagnostics Australia Pty. Ltd., Castle Hill, New South Wales, Australia). LDL cholesterol concentrations were calculated using the Friedewald formula [12]. Serum glucose was measured using an ultraviolet test with a fully automated analyser (Roche Hitachi 917).

\section{Statistics}

The primary outcome for this study was blood pressure measured as mean home blood pressure, measured twice daily (morning and evening) throughout each 7 day intervention, and mean 10 hour day-time ambulatory blood pressure, measured on day 7 of each intervention. If the true difference in mean blood pressure was $3.5 \mathrm{~mm} \mathrm{Hg}$, we estimated that 40 participants would be required to reject the null hypothesis that there is no difference with $80 \%$ power. Statistical analyses were performed using SPSS 21.0 (SPSS Inc, Chicago, IL). Baseline participant characteristics are presented as mean \pm SD and range. Categorical variables are summarized by number in each category. Results in text, tables and figures are presented as mean \pm SEM or mean (95\% CI). Outcome variables including salivary and plasma nitrate and nitrite, daytime ambulatory blood pressure and heart rate, PWV and AIx, were analyzed using ANCOVA, which compared post intervention values after adjustment for baseline. Home blood pressure was analysed using ANOVA. To investigate whether the effect of treatment on outcomes differed according to gender, age and BMI interaction terms were included in the models. A type-1 error rate of $\mathrm{P}<0.05$ was the level of significance used for all hypothesis testing. 


\section{Results}

\section{Baseline and descriptive data}

The study was conducted from May 2011 to November 2012. Forty-one participants were randomised and a total of thirty-eight participants (12 men and 26 women) completed the study (Figure 1). Data from all 38 participants who completed the study was available for analysis. Participant characteristics are shown in Table 1.

\section{Dietary nitrate, plasma and salivary nitrate and nitrite}

During the high nitrate diet intervention, participants increased their dietary nitrate intake from frozen spinach and fresh green leafy salad vegetables by $400 \pm 98 \mathrm{mg} / \mathrm{d}$ (Range: 225 to 695 $\mathrm{mg} / \mathrm{d}) .35$ of the 38 participants had an estimated increase in nitrate intake greater than $300 \mathrm{mg} / \mathrm{d}$. The frozen spinach provided to the participants contained $0.9 \mathrm{mg} / \mathrm{g}$ nitrate and the fresh green leafy salad vegetables provided to the participants was estimated to contain a mean of $1 \mathrm{mg} / \mathrm{g}$ nitrate.

Salivary and plasma nitrate and nitrite baseline and post intervention values are presented in Table 2. Salivary and plasma nitrate and nitrite concentrations were significantly increased $(\mathrm{P}<0.001)$ following the high nitrate diet compared to the low nitrate diet. The high nitrate diet resulted in a fivefold higher salivary nitrite, fourfold increase in salivary nitrate, a fourfold higher plasma nitrite and a sixfold increase in plasma nitrate (Figure 2).

We further investigated whether the effect of the intervention on salivary and plasma nitrate and nitrite might be influenced by gender, age and body mass index. However, inclusion of interaction terms in the models did not provide evidence of differential effects according to gender, age and body mass index $(\mathrm{P}>0.05)$. 


\section{Blood pressure and arterial stiffness}

Blood pressure and arterial stiffness baseline and post intervention values are presented in Table 2. The high nitrate diet compared to the low nitrate diet did not significantly alter systolic blood pressure, diastolic blood pressure or heart rate as measured by home blood pressure (Figure 3), ambulatory blood pressure (Figure 4) and office blood pressure (Figure 5).

No significant difference was observed in carotid-femoral pulse wave velocity (cfPWV) or augmentation index (AIx) at day 7 after the high nitrate diet compared to the low nitrate diet (Figure 6).

We further investigated whether the effect of the intervention on blood pressure might be influenced by gender, age and body mass index. However, inclusion of interaction terms in the models did not provide evidence of differential effects according to gender, age and body mass index $(\mathrm{P}>0.05)$. Change in plasma nitrite was not associated with change in ambulatory blood pressure (systolic blood pressure: $\mathrm{r}=-0.01, \mathrm{P}=0.96$; diastolic blood pressure: $\mathrm{r}=-0.01, \mathrm{P}=0.97$ ).

\section{Urinary sodium, potassium and creatinine}

Creatinine corrected urinary sodium $(\mathrm{Na} / \mathrm{Cr})$, potassium $(\mathrm{K} / \mathrm{Cr})$ and urinary sodium to potassium ratio $(\mathrm{Na} / \mathrm{K})$ at day 7 are provided in Table 3 . Creatinine corrected urinary sodium $(\mathrm{Na} / \mathrm{Cr})$ and potassium $(\mathrm{K} / \mathrm{Cr})$ excretion was significantly increased $(\mathrm{P}<0.05)$ at day 7 following the high nitrate diet compared to the low dietary nitrate. There was no significant difference $(\mathrm{P}=0.97)$ in the urinary sodium to potassium ratio $(\mathrm{Na} / \mathrm{K})$ between the two interventions. 


\section{Discussion}

The vascular effect of increased dietary nitrate from green leafy vegetables for 7 days in men and women with high normal blood pressure was assessed. Increases in salivary and plasma nitrate and nitrite provide evidence for a substantial increase in dietary nitrate intake and the potential for augmented NO formation with the high nitrate diet. These increases, however, did not result in lower blood pressure or reduced arterial stiffness.

The work presented here has a number of key strengths. To our knowledge, this is the first study to examine the vascular effect of increased nitrate intake from green leafy vegetables commonly consumed in the Western diet. This is the largest number of participants $(n=38)$ to date examining the effect of dietary nitrate on blood pressure. Results of clinical trials in normotensive individuals after nitrate intake have indicated the need to determine whether similar reductions in blood pressure would be observed in individuals with hypertension or at risk for hypertension. This is the first study to investigate dietary nitrate intake in individuals with high normal blood pressure. The study included rigorous assessment of blood pressure. Three clinically validated measurements were used to assess blood pressure. Home blood pressure monitoring was performed twice a day during the intervention. Ambulatory blood pressure, now considered the most reliable method of measuring blood pressure, and office blood pressure measurements were performed pre and post the intervention periods. Arterial stiffness was determined by carotidfemoral PWV, considered the gold standard for assessing central arterial stiffness.

Participants increased their dietary nitrate intake during the high nitrate diet by, on average, 400 mg per day, by consuming spinach and other green leafy vegetables. This is an easily achievable dose with previously demonstrated vascular benefits. Clinical studies, both acute and chronic, have observed beneficial effects on blood pressure and endothelial function in healthy individuals 
with nitrate doses ranging from 140 to $1500 \mathrm{mg}$ predominantly from beetroot juice and nitrate salts [13]. We have previously demonstrated vascular benefits after consumption of $182 \mathrm{mg}$ and $220 \mathrm{mg}$ nitrate from spinach $[7,8]$. This indicates that the intake in the current study of approximately $400 \mathrm{mg} /$ day is within the range previously demonstrated to have acute effects. Vegetables are the principal source of dietary nitrate contributing $80 \%$ of total nitrate intake [3]. The richest sources of nitrate are green leafy vegetables, such as lettuce and spinach, and beetroot. Other significant sources are drinking water $(<25 \%)$, which varies widely between populations, and red meat $(<10-15 \%)$ where nitrate/nitrite is added as a preservative. Nitrate intake varies considerably between individuals and populations. Individual intakes can range from less than $20 \mathrm{mg}$ to greater than $400 \mathrm{mg}$ per day [14, 15]. In Western populations nitrate intake has been estimated at $100 \mathrm{mg} /$ day [11]. Increasing nitrate intake to $400 \mathrm{mg}$ is easily achievable. Indeed, individuals who follow the Dietary Approaches to Stop Hypertension (DASH) diet can consume as much as $1000 \mathrm{mg} /$ day [3].

Salivary and plasma nitrate and nitrite was elevated following the high nitrate diet indicating an augmentation of the circulating pool of NO. Dietary nitrate can enhance the circulating pool of NO through the recently discovered enterosalivary nitrate-nitrite-NO pathway. The main sources of NO in the body are the enterosalivary nitrate-nitrite-NO pathway $[5,16,17]$ and the endogenous L-arginine-NOS pathway [18-22]. These two pathways are interconnected. Dietary nitrate, together with nitrate produced as an end product of NO metabolism from the L-arginine NOS pathway, enters the enterosalivary nitrate-nitrite-NO pathway. Here nitrate, actively absorbed from the plasma by the salivary glands, is concentrated in the saliva [23]. Salivary nitrate is reduced to nitrite by oral facultative anaerobic bacteria found in the deep clefts on the dorsal surface of the tongue [24]. Once swallowed a proportion of this nitrite is absorbed into the 
blood stream and may contribute to almost half of the total NO pool in the body (including plasma, red blood cells and tissues) and be the most important cell and tissue storage form of NO $[25,26]$. Nitrite to NO reduction can occur via a number of potential mechanisms including reactions with haemoglobin, myoglobin, xanthine oxidoreductase as well as acidic reduction [4].

The majority of studies examining the vascular effects of an acute or chronic dose of nitrate have been conducted in healthy normotensive individuals. To date only 4 studies have examined effects in those at risk for cardiovascular disease. Gilchrist et al investigating 14 day supplementation with $465 \mathrm{mg}$ nitrate from beetroot juice in 27 volunteers with type 2 diabetes (T2D) observed no effect on blood pressure or endothelial function [27]. Kenjale et al observed a significant decrease in diastolic blood pressure after a dose of $560 \mathrm{mg}$ nitrate from beetroot juice in 8 volunteers with peripheral artery disease [28]. Rammos et al demonstrated a reduction in systolic blood pressure and an improvement in endothelial function with $900 \mathrm{mg}$ sodium nitrate supplementation for 28 days in 11 elderly volunteers with a moderate risk of cardiovascular disease [29]. Recently Biswas et al observed a decrease in systolic blood pressure in prehypertensive patients after 30 days supplementation with a NO lozenge [30]. The ingredients of the lozenge include beetroot powder and hawthorn berry extract. The amount of nitrate, however, is not provided and the lozenge contained other phytochemicals from beetroot and hawthorn berry extract, such as flavonoids, which may have contributed to the benefit on blood pressure [31].

In this study of otherwise healthy participants with high normal blood pressure, and therefore at risk of developing hypertension, ambulatory, home monitored and office blood pressure as well as arterial stiffness, was unchanged following 7 days consumption of increased dietary nitrate from green leafy vegetables. These results do not concur with previous acute studies we have 
conducted with healthy volunteers as well as reports in the literature. Possible reasons for the apparent inconsistent results include the length of intervention, timing of measurements, volunteer cohort investigated, as well as new evidence suggesting a potential cross-talk between the enterosalivary nitrate-nitrite-NO pathway and the L-arginine-NOS pathway. The length of intervention in the current study was 7 days, a major difference with our previous studies which were acute $[7,8]$. While it is clear that an increase in nitrate can acutely reduce blood pressure, it is possible that the effect of regular consumption of nitrate on blood pressure is due to repeated acute effects. The timing of measurements in this current study would not have detected repeated acute effects. The volunteer cohort investigated here had high normal blood pressure. Elevated blood pressure and arterial stiffness are mutually causally related and indeed arterial stiffening is accelerated in subjects with high blood pressure [32]. Arterial stiffening is also strongly associated with increasing age [33]. The majority of volunteers in this study $(n=31)$ were over the age of 55. Elevated blood pressure, increased arterial stiffness and age are independently associated with decreased NO production $[34,35]$. Evidence suggests that the enterosalivary nitrate-nitrite-NO pathway is a backup pathway to the L-arginine-NOS pathway. However, the combination of elevated blood pressure, age of the cohort as well as increased arterial stiffness, all associated with decreased NO production, may have diminished the vascular benefit of dietary nitrate consumption. Whether a longer dietary nitrate intake in this cohort would have vascular benefits remains to be determined. There is also new evidence to suggest that the enterosalivary nitrate-nitrite-NO pathway and the L-arginine-NOS pathway are linked [36]. Increasing NO through one pathway could down-regulate the other pathway resulting in a net zero effect. This is referred to as cross-talk between the pathways and has yet to be confirmed in human studies. 
While volunteers were required to maintain usual diet and lifestyle, the increase in green leafy vegetables could have impacted other aspects of their diet such as meat intake. Urinary creatinine, however, was similar between the low and high dietary nitrate interventions. The lack of a blood pressure lowering response may relate to an increase in sodium intake, although potassium intake was also increased. It is possible that unintended dietary changes could have influenced the blood pressure effects of increased intake of green leafy vegetables.

It is also possible that there are differential effects according to gender, age and body mass index, however the study was not designed or powered to detect an interaction or do a stratified analysis.

In conclusion, we found that regular consumption (7 days) of increased dietary nitrate from green leafy vegetables had no effect on blood pressure or arterial stiffness in men and women with high-normal blood pressure. Contrary to evidence from clinical trials with healthy individuals, this study suggests that increasing intake of vegetables rich in dietary nitrate may not be an effective antihypertensive strategy in individuals with high normal blood pressure and therefore at risk for developing hypertension. This study, however, was only conducted over 1 week. Future studies are required to confirm this finding with a long-term dietary nitrate intake intervention and in other populations at risk for cardiovascular disease. 


\section{Acknowledgements}

The authors thank Ms Adeline Indrawan and Mrs Lisa Rich for technical assistance. The authors thank Logan Farms for supplying the spinach. J.M. Hodgson acknowledges the support of a National Health and Medical Research Council Senior Research Fellowship. N.C. Ward acknowledges the support of a Royal Perth Hospital Medical Research Foundation

Fellowship/UWA. This work was funded by the National Health and Medical Research Council. 


\section{References}

[1] Joshipura, K. J.; Ascherio, A.; Manson, J. E.; Stampfer, M. J.; Rimm, E. B.; Speizer, F.

E.; Hennekens, C. H.; Spiegelman, D.; Willett, W. C. Fruit and vegetable intake in relation to risk of ischemic stroke. Jama 282:1233-1239; 1999.

[2] Joshipura, K. J.; Hu, F. B.; Manson, J. E.; Stampfer, M. J.; Rimm, E. B.; Speizer, F. E.; Colditz, G.; Ascherio, A.; Rosner, B.; Spiegelman, D.; Willett, W. C. The effect of fruit and vegetable intake on risk for coronary heart disease. Ann. Intern. Med. 134:1106-1114; 2001.

[3] Hord, N. G.; Tang, Y.; Bryan, N. S. Food sources of nitrates and nitrites: the physiologic context for potential health benefits. Am. J. Clin. Nutr. 90:1-10; 2009.

[4] Lundberg, J. O.; Gladwin, M. T.; Ahluwalia, A.; Benjamin, N.; Bryan, N. S.; Butler, A.;

Cabrales, P.; Fago, A.; Feelisch, M.; Ford, P. C. Nitrate and nitrite in biology, nutrition and therapeutics. Nat. Chem. Biol. 5:865-869; 2009.

[5] Webb, A. J.; Patel, N.; Loukogeorgakis, S.; Okorie, M.; Aboud, Z.; Misra, S.; Rashid, R.; Miall, P.; Deanfield, J.; Benjamin, N.; MacAllister, R.; Hobbs, A. J.; Ahluwalia, A. Acute blood pressure lowering, vasoprotective, and antiplatelet properties of dietary nitrate via bioconversion to nitrite. Hypertension 51:784-790; 2008.

[6] Bondonno, C. P.; Croft, K. D.; Ward, N. C.; Considine, M. J.; Hodgson, J. M. Dietary flavonoids and nitrate: effects on nitric oxide and vascular function. Nutr Rev doi:

10.1093/nutrit/nuu014; 2014.

[7] Bondonno, C. P.; Yang, X.; Croft, K. D.; Considine, M. J.; Ward, N. C.; Rich, L.;

Puddey, I. B.; Swinny, E.; Mubarak, A.; Hodgson, J. M. Flavonoid-rich apples and nitrate-rich spinach augment nitric oxide status and improve endothelial function in healthy men and women: a randomized controlled trial. Free Radic. Biol. Med. 52:95-102; 2012. 
[8] Liu, A. H.; Bondonno, C. P.; Croft, K. D.; Puddey, I. B.; Woodman, R. J.; Rich, L.; Ward, N. C.; Vita, J. A.; Hodgson, J. M. Effects of a nitrate-rich meal on arterial stiffness and blood pressure in healthy volunteers. Nitric Oxide 35:123-130; 2013.

[9] Sobko, T.; Marcus, C.; Govoni, M.; Kamiya, S. Dietary nitrate in Japanese traditional foods lowers diastolic blood pressure in healthy volunteers. Nitric Oxide 22:136-140; 2010. [10] Yang, X.; Bondonno, C. P.; Indrawan, A.; Hodgson, J. M.; Croft, K. D. An improved mass spectrometry-based measurement of NO metabolites in biological fluids. Free Rad Biol Med 56:1-8; 2013.

[11] Griesenbeck, J. S.; Steck, M. D.; Huber Jr, J. C.; Sharkey, J. R.; Rene, A. A.; Brender, J. D. Development of estimates of dietary nitrates, nitrites, and nitrosamines for use with the short willet food frequency questionnaire. Nutr J 8:16-16; 2009.

[12] Friedewald, W. T.; Levy, R. I.; Fredrickson, D. S. Estimation of the concentration of lowdensity lipoprotein cholesterol in plasma, without use of the preparative ultracentrifuge. Clin. Chem. 18:499-502; 1972.

[13] Bondonno, C. P.; Croft, K. D.; Considine, M. J.; Hodgson, J. M. Dietary flavonoids and nitrate: effects on nitric oxide and vascular function. Nutr. Rev. In press.; 2014.

[14] Petersen, A.; Stoltze, S. Nitrate and nitrite in vegetables on the Danish market: content and intake. Food Addit. Contam. 16:291 - 299; 1999.

[15] Ysart, G.; Miller, P.; Barrett, G.; Farrington, D.; Lawrance, P.; Harrison, N. Dietary exposures to nitrate in the UK. Food Addit. Contam. 16:521 - 532; 1999.

[16] Govoni, M.; Jansson, E. A.; Weitzberg, E.; Lundberg, J. O. The increase in plasma nitrite after a dietary nitrate load is markedly attenuated by an antibacterial mouthwash. Nitric Oxide 19:333-337; 2008. 
[17] Petersson, J.; Carlström, M.; Schreiber, O.; Phillipson, M.; Christoffersson, G.; Jägare, A.; Roos, S.; Jansson, E. Å.; Persson, A. E. G.; Lundberg, J. O.; Holm, L. Gastroprotective and blood pressure lowering effects of dietary nitrate are abolished by an antiseptic mouthwash. Free Radic. Biol. Med. 46:1068-1075; 2009.

[18] Alderton, W. K.; Cooper, C. E.; Knowles, R. G. Nitric oxide synthases: structure, function and inhibition. Biochem. J. 357:593; 2001.

[19] Bredt, D. S. Endogenous nitric oxide synthesis: biological functions and pathophysiology. Free Radical Res. 31:577-596; 1999.

[20] Bredt, D. S. Nitric oxide signaling specificity-the heart of the problem. J. Cell Sci. 116:915; 2003.

[21] Jin, R. C.; Loscalzo, J. Vascular nitric oxide: formation and function. J. Blood Med. 1:147-162; 2010.

[22] Liu, V. W. T.; Huang, P. L. Cardiovascular roles of nitric oxide: A review of insights from nitric oxide synthase gene disrupted mice Cardiovasc. Res. 77:19-29; 2008.

[23] Spiegelhalder, B.; Eisenbrand, G.; Preussmann, R. Influence of dietary nitrate on nitrite content of human saliva: Possible relevance to in vivo formation of N-nitroso compounds. Food Cosmet. Toxicol. 14:545-548; 1976.

[24] Gladwin, M. T.; Schechter, A. N.; Kim-Shapiro, D. B.; Patel, R. P.; Hogg, N.; Shiva, S.; Cannon, R. O., 3rd; Kelm, M.; Wink, D. A.; Espey, M. G.; Oldfield, E. H.; Pluta, R. M.; Freeman, B. A.; Lancaster, J. R., Jr.; Feelisch, M.; Lundberg, J. O. The emerging biology of the nitrite anion. Nat. Chem. Biol. 1:308-314; 2005.

[25] Larsen, F. J.; Ekblom, B.; Sahlin, K.; Lundberg, J. O.; Weitzberg, E. Effects of dietary nitrate on blood pressure in healthy volunteers. N. Engl. J. Med. 355:2792-2793; 2006. 
[26] Lundberg, J. O.; Govoni, M. Inorganic nitrate is a possible source for systemic generation of nitric oxide. Free Radic. Biol. Med. 37:395-400; 2004.

[27] Gilchrist, M.; Winyard, P. G.; Aizawa, K.; Anning, C.; Shore, A.; Benjamin, N. k. Free Rad Biol Med 60:89-97; 2013.

[28] Kenjale, A. A.; Ham, K. L.; Stabler, T.; Robbins, J. L.; Johnson, J. L.; VanBruggen, M.;

Privette, G.; Yim, E.; Kraus, W. E.; Allen, J. D. Dietary nitrate supplementation enhances exercise performance in peripheral arterial disease. J Appl Physiol 110:1582-1591; 2011.

[29] Rammos, C.; Hendgen-Cotta, U. B.; Sobierajski, J.; Bernard, A.; Kelm, M.; Rassaf, T.

Dietary nitrate reverses vascular dysfunction in old adults with moderately increased cardiovascular risk. J Am Coll Cardio 63:1584-1585; 2013.

[30] Biswas, O. S.; Gonzalez, V. R.; Schwarz, E. R. Effects of an oral nitric oxide supplement on functional capacity and blood pressure in adults with prehypertension. $J$ Cardiovasc Pharmacol Ther; 2014.

[31] Hodgson, J.; Puddey, I. B.; Woodman, R. J.; et al. Effects of black tea on blood pressure: A randomized controlled trial. Arch. Intern. Med 172:186-188; 2012.

[32] Yambe, M.; Tomiyama, H.; Yamada, J.; Koji, Y.; Motobe, K.; Shiina, K.; Yamamoto, Y.; Yamashina, A. Arterial stiffness and progression to hypertension in Japanese male subjects with high normal blood pressure. J Hypertens 25:87-93; 2007.

[33] Lee, H.-Y.; Oh, B.-H. Aging and arterial stiffness. Circ J 74:2257; 2010.

[34] Taddei, S.; Virdis, A.; Ghiadoni, L.; Salvetti, G.; Bernini, G.; Magagna, A.; Salvetti, A. Age-related reduction of NO availability and oxidative stress in humans. Hypertension 38:274279; 2001. 
[35] Lyamina, N. P.; Dolotovskaya, P. V.; Lyamina, S.; Malyshev, I. Y.; Manukhina, E. B. Nitric oxide production and intensity of free radical processes in young men with high normal and hypertensive blood pressure. Med Sci Monit 9:CR304-310; 2003.

[36] Carlström, M.; Liu, M.; Yang, T.; Zollbrecht, C.; Huang, L.; Peleli, M.; Borniquel, S.; Kishikawa, H.; Hezel, M.; Persson, A. E. G. Cross-talk between nitrate-nitrite-NO and NO synthase pathways in control of vascular NO homeostasis. Antioxid Redox Sign

doi:10.1089/ars.2013.5481; 2013. 


\section{Figure legends}

Figure 1: $\quad$ Participant flow from recruitment through screening and randomisation to trial completion.

Figure 2: $\quad$ Baseline adjusted post intervention concentrations of (A) salivary nitrite, (B) salivary nitrate, (C) plasma nitrite and (D) plasma nitrate for the low nitrate diet and high nitrate diet interventions. Effects were analysed using ANCOVA in SPSS. Results are expressed as mean and standard error.

Figure 3: $\quad$ Effects of a 7 day high nitrate diet intervention compared with a 7 day low nitrate diet intervention in individuals with high normal blood pressure $(n=38)$ on home blood pressure monitored (A) systolic blood pressure from day 1 evening (D1E) to day 5 evening (D5E) of the intervention period, (B) mean baseline adjusted systolic blood pressure, (C) diastolic blood pressure from D1E to D5E of the intervention period, (D) mean baseline adjusted diastolic blood pressure, (E) heart rate from D1E to D5E of the intervention period and (F) mean baseline adjusted heart rate. Effects were analysed using ANOVA in SPSS. Results are expressed as mean and standard error. The $\mathrm{P}$ value is post intervention difference between low nitrate and high nitrate diets after adjustment for baseline values.

Figure 4: $\quad$ Effects of a 7 day high nitrate diet intervention compared with a 7 day low nitrate diet intervention in individuals with high normal blood pressure $(n=38)$ on 10 hour day-time ambulatory (A) mean hourly systolic blood pressure, (B) mean baseline adjusted systolic blood pressure post intervention, (C) mean hourly diastolic blood pressure, (D) mean baseline adjusted diastolic blood pressure post intervention, 
(E) mean hourly heart rate and (F) mean baseline adjusted heart rate post intervention. Effects were analysed using ANCOVA in SPSS. Results are expressed as mean and standard error. The P value is post intervention difference between low nitrate and high nitrate diets after adjustment for baseline values.

Figure 5: $\quad$ Effects of a 7 day high nitrate diet intervention compared with a 7 day low nitrate diet intervention in individuals with high normal blood pressure $(\mathrm{n}=38)$ on office (A) systolic blood pressure, (B) diastolic blood pressure and (C) heart rate. Effects were analysed using ANCOVA in SPSS. Results are expressed as mean and standard error. The P value is post intervention difference between low nitrate and high nitrate diets after adjustment for baseline values.

Figure 6: Effects of a 7 day high nitrate diet intervention compared with a 7 day low nitrate diet intervention in individuals with high normal blood pressure (n=38) on (A) carotid-femoral pulse wave velocity, cfPWV; (B) augmentation index calculated as the ratio between augmented pressure and pulse pressure, AIx (AP/PP); (C) augmentation index calculated as the ratio between first systolic peak (P1) and second systolic peak (P2), AIx (P1/P2) and (D) augmentation index normalised for heart rate of 75 bpm, AIx@75 .Effects were analysed using ANCOVA in SPSS. Results are expressed as mean and standard error. The $\mathrm{P}$ value is post intervention difference between low nitrate and high nitrate diets after adjustment for baseline values. 
Table1. Baseline characteristics of study subjects ( $n=38$; males $n=12$; females $n=26$ )

\begin{tabular}{|c|c|c|c|}
\hline & Range & Mean & Standard Deviation \\
\hline Age (years) & $38-70$ & 60.6 & 7.0 \\
\hline Height (cm) & $147.5-181.5$ & 168.0 & 8.6 \\
\hline Weight (kg) & $44.8-108.2$ & 77.1 & 13.9 \\
\hline Body mass index $\left(\mathrm{kg} / \mathrm{m}^{2}\right)$ & $20.6-37.0$ & 27.1 & 3.8 \\
\hline Systolic blood pressure (mm Hg) & $120-140$ & 130.0 & 5.8 \\
\hline Diastolic blood pressure (mm Hg) & $62-90$ & 75.9 & 6.8 \\
\hline Heart rate (bpm) & $50-84$ & 63.9 & 8.4 \\
\hline Total cholesterol (mmol/L) & $3.3-6.8$ & 5.4 & 0.8 \\
\hline HDL cholesterol (mmol/L) & $0.9-2.8$ & 1.4 & 0.4 \\
\hline Triglycerides $(\mathrm{mmol} / \mathrm{L})^{\mathrm{a}}$ & $0.5-2.0$ & 1.0 & $(0.9,1.1)$ \\
\hline Glucose $(\mathrm{mmol} / \mathrm{L})$ & $4.7-6.2$ & 5.3 & 0.4 \\
\hline
\end{tabular}

${ }^{\mathrm{a}}$ Geometric mean (95\% confidence interval) 
Table 2 : Salivary and plasma nitrate/nitrite, blood pressure, heart rate, and arterial stiffness measures at baseline and post intervention for low and high nitrate diets ${ }^{\mathrm{a}}$

\begin{tabular}{|c|c|c|c|c|c|}
\hline & & \multicolumn{2}{|c|}{ Low nitrate diet } & \multicolumn{2}{|c|}{ High nitrate diet } \\
\hline & & Baseline $^{b}$ & Intervention $^{c}$ & Baseline $^{\text {b }}$ & Intervention ${ }^{c}$ \\
\hline \multirow[t]{2}{*}{ Nitrate } & Salivary nitrate $(\mu \mathrm{mol} / \mathrm{L})$ & $263.0(239.0)$ & $231.9(182.3)$ & $204.7(149.4)$ & $1334.7(706.9)$ \\
\hline & Plasma nitrate $(\mu \mathrm{mol} / \mathrm{L})$ & $26.2(13.2)$ & $24.5(9.9)$ & $23.4(10.5)$ & $151.8(62.0)$ \\
\hline Nitrite & Plasma nitrite $(\mu \mathrm{mol} / \mathrm{L})$ & $2.7(4.0)$ & $2.0(1.5)$ & $2.0(1.5)$ & $8.0(6.5)$ \\
\hline \multirow[t]{2}{*}{ Home BP } & SBP (mm Hg) & $122.6(8.5)$ & $122.4(9.0)$ & $121.6(8)$ & $122.3(8.4)$ \\
\hline & DPB (mm Hg) & $72.5(8.3)$ & $72.3(8.4)$ & $71.7(6.8)$ & $72.5(8.2)$ \\
\hline \multirow{2}{*}{ Ambulatory BP } & DPB (mm Hg) & $76.5(8.6)$ & $76.3(.07)$ & $76.1(7.9)$ & $77.3(8.3)$ \\
\hline & HR (bpm) & $73.2(8.0)$ & $73.7(8.0)$ & $73.6(9.2)$ & $74.9(10.2)$ \\
\hline \multirow[t]{3}{*}{ Office BP } & SBP (mm Hg) & $130.3(12.2)$ & $130.0(10.8)$ & $129.0(12.5)$ & $126.2(11.8)$ \\
\hline & DPB (mm Hg) & $72.4(7.3)$ & $70.5(6.4)$ & $69.1(7.7)$ & $70.7(8.8)$ \\
\hline & HR (bpm) & $61.4(8.9)$ & $61.5(8.3)$ & $61.6(8.5)$ & $62.7(7.3)$ \\
\hline
\end{tabular}




$\begin{array}{ccccc}\text { AIx (AP/PP) (\%) } & \\ & 32.4(10.3) & 31.4(10.7) & 32.1(9.2) & 32.4(9.6) \\ \text { AIx (P1/P2) (\%) } & 152.0(27.4) & 149.4(26.2) & 149.9(20.2) & 150.8(21.3) \\ \text { AIx@75 (\%) } & 26.4(10.1) & 26.4(9.4) & 26.1(8.6) & 26.8(9.3)\end{array}$

${ }^{\text {a }}$ Results are expressed as means (SD).

${ }^{\mathrm{b}}$ Baseline: After 7 days of following a low nitrate diet;

${ }^{\mathrm{c}}$ Intervention: after 7 days of following either a low nitrate diet (same as the diet during baseline) or high nitrate diet

${ }^{\mathrm{d}}$ cfPWV - carotid-femoral pulse wave velocity.

${ }^{\mathrm{e}} \mathrm{AIx}(\mathrm{AP} / \mathrm{PP})$ - augmentation index (AIx) calculated as the ratio between augmented pressure (AP) and pulse pressure (PP).

${ }^{\mathrm{f}}$ AIx (P1/P2) - augmentation index (AIx) calculated as the ratio between first systolic peak (P1) and second systolic peak (P2).

g AIx@75 - augmentation index (AIx) normalised for heart rate of 75 bpm. 
Table 3: $\quad$ Twenty four hour urinary sodium, potassium, creatinine and creatinine corrected urinary sodium $(\mathrm{Na} / \mathrm{Cr})$, potassium $(\mathrm{K} / \mathrm{Cr})$ and urinary sodium to potassium ratio $(\mathrm{Na} / \mathrm{K})$ at day 7 for low and high nitrate diet ${ }^{\mathrm{a}}$.

\begin{tabular}{cccc}
\hline & Low nitrate diet & High nitrate diet & P-value \\
\hline Urinary sodium (mmol/d) & $110.1(40.9)$ & $136.2(46.0)$ & 0.004 \\
$\begin{array}{c}\text { Urinary potassium } \\
(\mathrm{mmol} / \mathrm{d})\end{array}$ & $72.2(27.9)$ & $87.8(25.4)$ & 0.001 \\
$\begin{array}{c}\text { Urinary creatinine } \\
(\text { mmol/d) }\end{array}$ & $10.4(3.8)$ & $10.5(3.2)$ & 0.9 \\
$\begin{array}{c}\text { Urinary sodium } \\
(\text { mmol/d/mmol creatinine })\end{array}$ & $11.4(5.2)$ & $13.6(5.2)$ & 0.05 \\
$\begin{array}{c}\text { Urinary potassium } \\
(\text { mmol/d/mmol creatinine })\end{array}$ & $7.3(2.4)$ & $8.9(3.6)$ & 0.001 \\
$\begin{array}{c}\text { Urinary sodium-to- } \\
\text { potassium ratio }\end{array}$ & $1.7(0.8)$ & $1.7(0.8)$ & 0.97 \\
\hline
\end{tabular}

${ }^{\mathrm{a}}$ Results are expressed as means (SD). 
Figure 1.

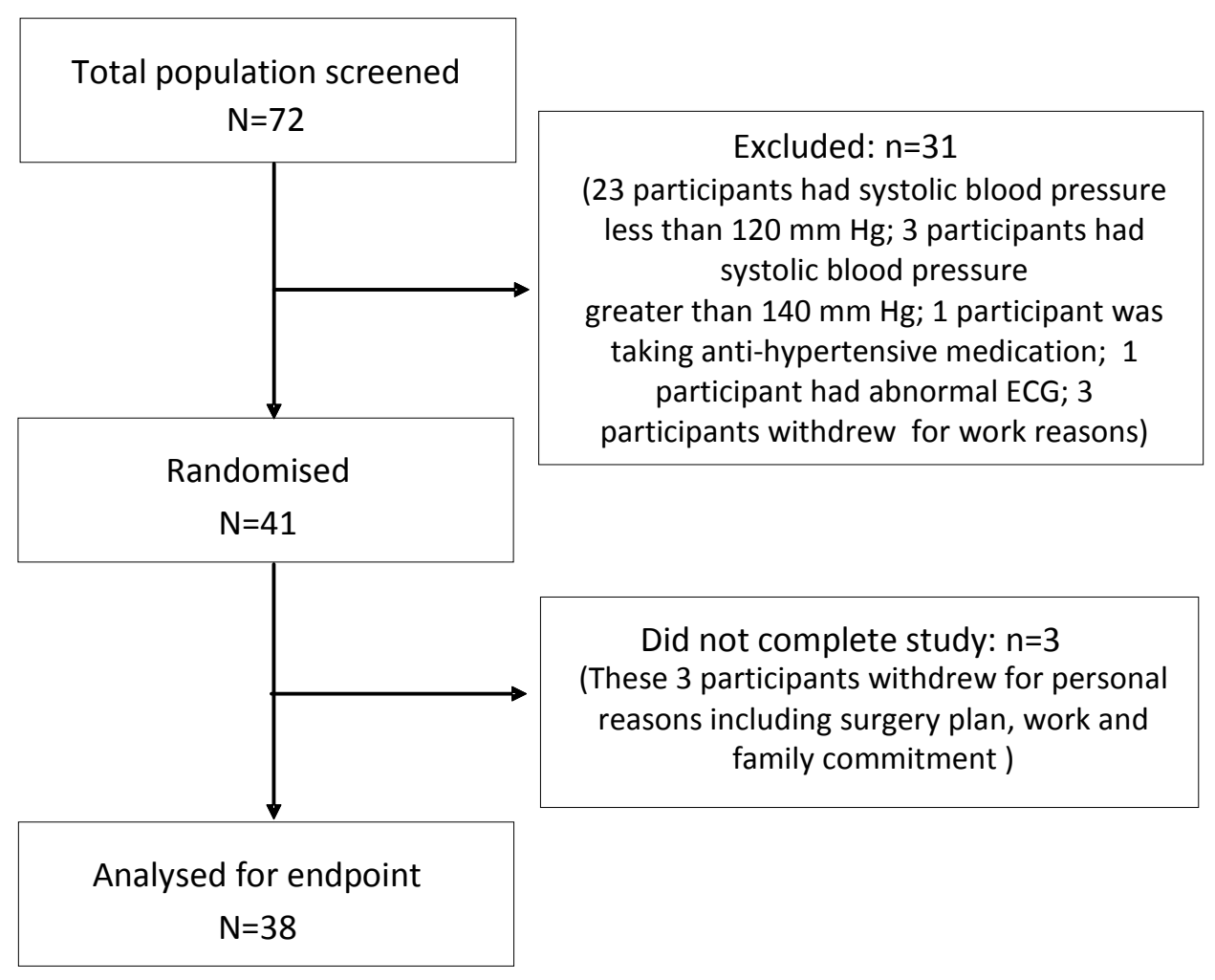


Figure 2.
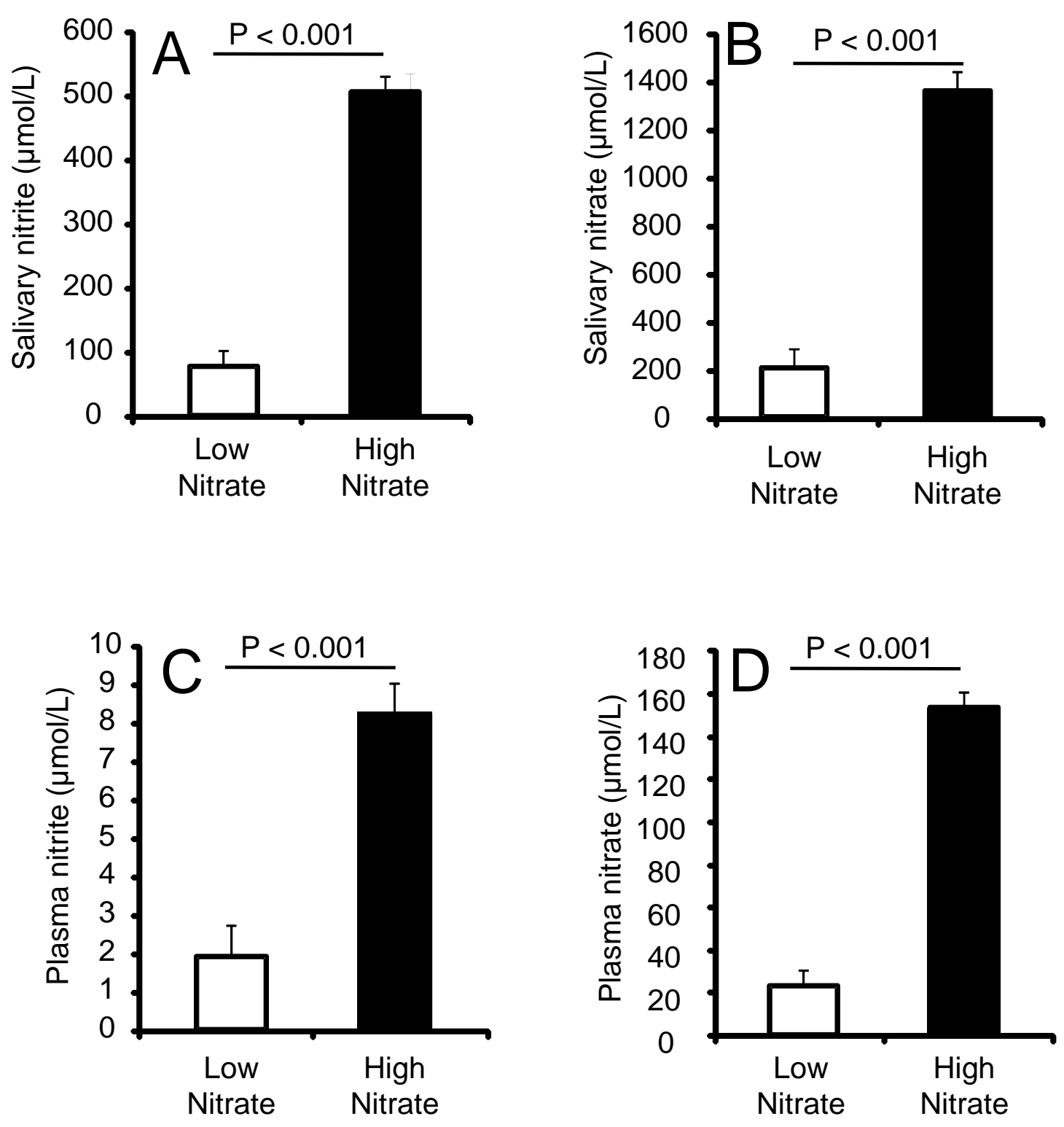
Figure 3.

Figure 4.
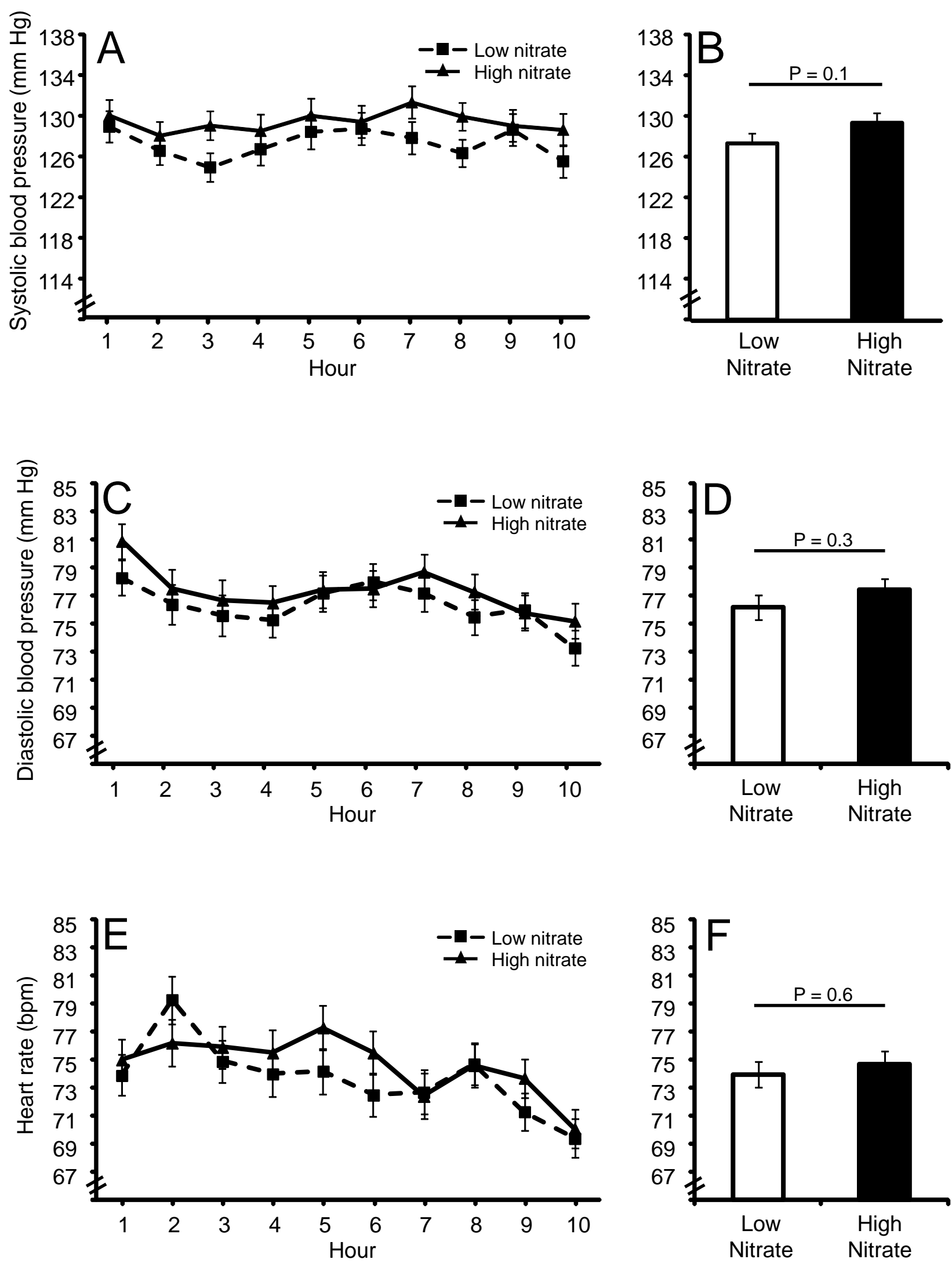
Figure 5.
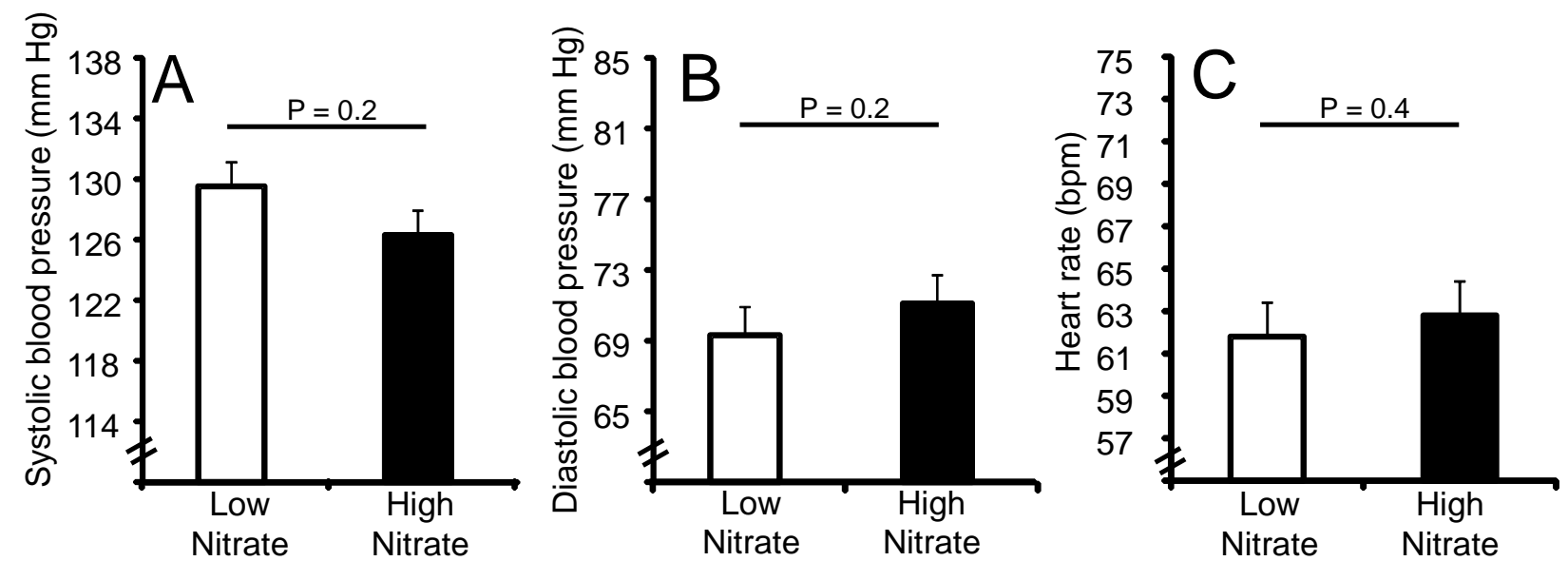
Figure 6.
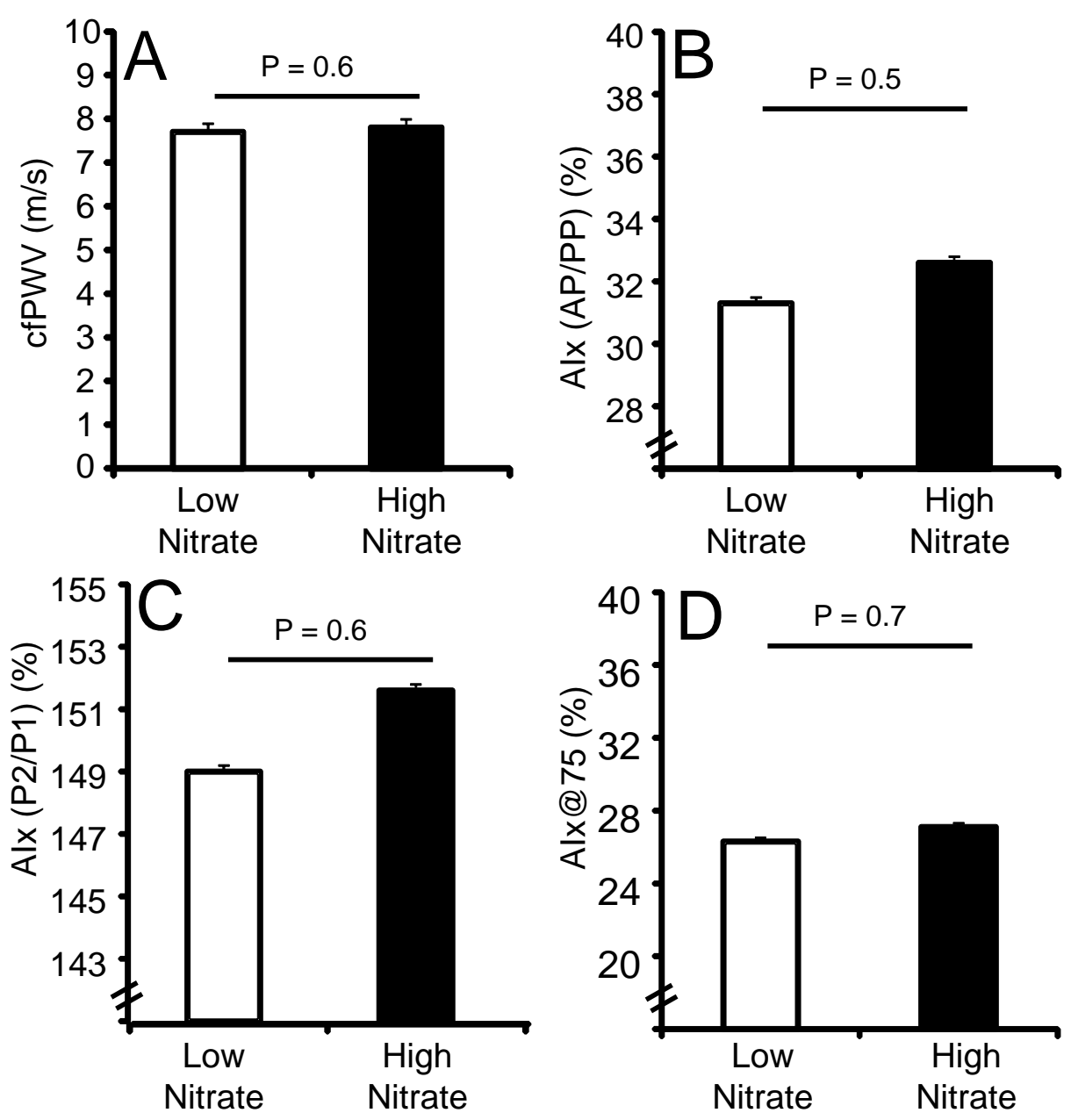